\title{
COMPLETE POSITIVITY AND THE NEUTRAL KAON SYSTEM
}

\author{
FABIO BENATTI \\ Dipartimento di Fisica Teorica, Università di Trieste \\ and I.N.F.N., Sezione di Trieste \\ Strada Costiera 11, 34100 Trieste, Italy \\ E-mail: benatti@ts.infn.it \\ ROBERTO FLOREANINI \\ I.N.F.N., Sezione di Trieste \\ Strada Costiera 11, 34100 Trieste, Italy \\ E-mail: florean@ts.infn.it
}

\begin{abstract}
New experiments on neutral K-mesons might turn out to be promising tests of the hypothesis of Complete Positivity in the physics of open quantum systems. In particular, a consistent dynamical description of correlated neutral kaons seems to ask for Complete Positivity.

1. Introduction. Quantum dynamical semigroups provide a useful framework for the description of quite a variety of quantum open systems $(S)([1,2])$. From a general point of view, the latter are considered as subsystems interacting with a suitable environment $(E)$. If the coupling between $S$ and $E$ is sufficiently weak, the effective evolution of $S$ is expected to be irreversible and free from complicated feed-back and memory effects. Under these hypothesis, via weak-coupling limits techniques, one can indeed deduce an effective Markoffian dynamics ([1-4]). That is, the space of states $\rho$ (density matrices) of $S$ is transformed into itself by a one-parameter family $\gamma_{t}: \rho \mapsto \gamma_{t}[\rho]$ of linear maps satisfying $\gamma_{s} \circ \gamma_{t}=\gamma_{t+s}$ for $s, t \geq 0$ and preserving the positivity of any initial state $\rho$.

However, it turns out that the dynamical maps $\gamma_{t}$ obtained in this way enjoy the stronger property of being completely positive $([5,6])$. That is, they remain positive when naturally extended from linear transformations on the state-space of $S$ to linear transformations on the state-space of the system $S$ coupled with any finite-level system $E_{n}$ of arbitrary dimension $n$ :
\end{abstract}

$$
\gamma_{t} \quad \text { on } S \longrightarrow \gamma_{t} \otimes 1_{n} \text { on } S+E_{n} .
$$

1991 Mathematics Subject Classification: 82C10, 81V15.

The paper is in final form and no version of it will be published elsewhere. 
(Complete Positivity is usually defined for linear transformations on observables. Via duality, the property is then extended to linear maps on the state-space as formulated in (1.1).)

The importance of complete positivity for addressing the description of quantum measurement processes has been pointed out in [6] and the consequences on the generators of quantum dynamical semigroups in [7,8].

If we ask that the effective dynamics of a finite-level quantum open system $S$ be described by a probability preserving quantum dynamical semigroup, the evolution equation satisfied by the states of $S$ has the typical Lindblad form

$$
\frac{\partial}{\partial t} \gamma_{t}[\rho]=-i\left[H, \gamma_{t}[\rho]\right]-\frac{1}{2} \sum_{j}\left\{A_{j}^{\dagger} A_{j} \gamma_{t}[\rho]+\gamma_{t}[\rho] A_{j}^{\dagger} A_{j}\right\}+\sum_{j} A_{j} \gamma_{t}[\rho] A_{j}^{\dagger}
$$

From (1.2) it follows that the request of complete positivity forced upon the dynamics of a quantum open system has striking consequences on the phenomenological parameters governing the effective description: typically, relaxation times satisfy certain inequalities $([1])$.

The question naturally arises whether complete positivity is really necessary. After all, it results as a constraint from the coupling of $S$ with a dynamically totally independent external system $E_{n}$. Why should this apparently trivial "interaction" have dynamical effects on $S$ which, in itself, is not at all influenced by $E_{n}$ but for the correlations built in the common state of $S+E_{n}([9])$ ?

In effect, renouncing complete positivity asks for some price to be paid, in terms either of internal inconsistencies or of lack of generality in defining a consistent reduced dynamics ([10]). Indeed, generic compound states of $S+E_{n}$ are entangled, that is they cannot be put in the "separated" form

$$
\rho_{S+E_{n}}=\sum_{i, j} \lambda_{i j} \rho_{S}^{i} \otimes \rho_{E_{n}}^{j}
$$

where $\rho_{S}^{i}$, respectively $\rho_{E_{n}}^{j}$, are states of $S$, respectively $E_{n}$ and $\lambda_{i j}$ are positive weights. Then (compare also $[11,12]$ ),

1. If $\gamma_{t}$ is completely positive on $S$, all states of $S+E_{n}$, whether entangled or not, are transformed into states of $S+E_{n}$ by $\gamma_{t} \otimes 1_{n}$, for all $n$.

2. If $\gamma_{t}$ is just positive on $S$, there exist an $E_{n}$ and a non-separable state $\rho_{S+E_{n}}^{*}$ of $S+E_{n}$ such that the spectrum of $\gamma_{t} \otimes 1_{n}\left[\rho_{S+E_{n}}^{*}\right]$ is not positive any more and $\gamma_{t} \otimes 1_{n}\left[\rho_{S+E_{n}}^{*}\right]$ cannot be thus interpreted as a state of $S+E_{n}$.

Whether the latter fact may have any importance for physics is, at first sight, doubtful in view of the physical triviality of the coupling (1.1) and the possible physical hawkwardness of the pathological state $\rho_{S+E_{n}}^{*}$. Nevertheless, by considering the system of neutral $K$-mesons (kaons) ([13]), in the following we will present arguments that would illustrate points 1 . and 2 . above.

The observation that the quantum fluctuations of the gravitational field at Planck's scale originate loss of quantum coherence $([14,15])$ transforming pure states into mixtures $([16,17])$ was one of the physical motivations to describe the neutral kaon system as an open quantum system $([18,19])$. This approach essentially amounts to postulating for 
unstable particles like kaons a dynamics not of the standard (Weisskopf-Wigner) form. In turn, such non-standard time-evolution would induce violations of the symmetries under Charge-Parity $(C P-)$ and even under $C P$ plus Time-reversal $(C P T-)$ transformations. Interestingly enough, the consequences of such an hypothesis could be compared with existing experimental data.

In [20] it was observed that the dissipative semigroup dynamics of $[18,19]$ cannot be completely positive in general. Instead, in [20-23] the $K^{0}-\overline{K^{0}}$ system has been treated as a quantum open system evolving according to a quantum dynamical semigroup of completely positive maps. Indeed, the coupling between the kaon system and the quantum gravitational background can be certainly supposed to be weak enough to justify an effective reduced dynamics with such a property. Moreover, one can conceive different mechanisms $([24,25])$ than quantum gravity as responsible for the $K^{0}-\overline{K^{0}}$ dissipative open dynamics. In such a case quantum dynamical semigroups provide a rather flexible and rich framework for a convenient description of the kaon decay. The dissipative modification of the standard Weisskopf-Wigner evolution equation introduces six new phenomenological parameters $a, b, c, \alpha, \beta$ and $\gamma$ that must obey certain inequalities. These inequalities can, in principle, be checked by using the available experimental data on the neutral kaon system.

Unfortunately ([23]), the accuracies of experimental data available at present are too poor to perform such a test. However, planned future experiments at the so-called $\phi$-factories involving pair of correlated neutral kaons are expected to provide much better accuracies $([26,27])$. Moreover, the natural extension of the quantum open system description to the physics of the entangled kaons at a $\phi$-factory points to complete positivity as a necessary property against the appearance of unphysical negative eigenvalues in the spectrum of the two-kaon states $([21,22])$.

2. Neutral kaon system. The evolution and decay of the neutral kaons $K^{0}$ and $\overline{K^{0}}$ is usually described by means of a two-dimensional Hilbert space ([13]). The kaon states $\left|K^{0}\right\rangle$ and $\left|\overline{K^{0}}\right\rangle$ with strangeness quantum number +1 , respectively -1 are exchanged one into the other under a $C P$ (Charge-Parity) transformation. The $C P$ (Charge-Parity) eigenstates

$$
\left|K_{1}\right\rangle=\frac{1}{\sqrt{2}}\left[\left|K^{0}\right\rangle+\left|\overline{K^{0}}\right\rangle\right], \quad\left|K_{2}\right\rangle=\frac{1}{\sqrt{2}}\left[\left|K^{0}\right\rangle-\left|\overline{K^{0}}\right\rangle\right]
$$

will be used as a Hilbert space basis and mixed kaon states (density matrices) will be accordingly represented as:

$$
\rho=\left(\begin{array}{cc}
\rho_{0}+\rho_{3} & \rho_{1}-i \rho_{2} \\
\rho_{1}+i \rho_{2} & \rho_{0}-\rho_{3}
\end{array}\right) .
$$

Because they interact weakly, neither strangeness nor $C P$-invariance is preserved and the above states decay in time. In the standard quantum mechanical description, the decay properties of the $K^{0}-\overline{K^{0}}$ system are described by an effective (Weisskopf-Wigner) Hamiltonian

$$
H=M-\frac{i}{2} \Gamma
$$


where $M$ and $\Gamma$ are positive $2 \times 2$ matrices, $\Gamma$ characterizing the decaying properties of the system.

As explained in the introduction, we will abandon the standard description and treat neutral kaons as open quantum systems evolving in time according to a semigroup of dynamical maps generated by a Weisskopf-Wigner term $-i H \rho(t)+i \rho(t) H^{\dagger}$ plus a dissipative one $L_{D}[\rho(t)]$ of Lindblad form:

$$
\begin{gathered}
\frac{\partial}{\partial t} \rho(t)=-i H \rho(t)+i \rho(t) H^{\dagger}+L_{D}[\rho(t)], \\
L_{D}[\rho(t)]=-\frac{1}{2}(R \rho(t)+\rho(t) R)+\sum_{j} A_{j} \rho(t) A_{j}^{\dagger},
\end{gathered}
$$

where $R:=\sum_{j} A_{j}^{\dagger} A_{j}$ and $L_{D}[\rho]$ are well-defined $2 \times 2$ matrices for any given state $\rho$.

A part from being probability decreasing $(\mathrm{d} / \mathrm{d} t \operatorname{Tr} \rho(t)=-\operatorname{Tr}(\rho(t) \Gamma) \leq 0),(2.4)$ generates a quantum dynamical semigroup $([1,3,7])$, namely the linear maps $\gamma_{t}: \rho \mapsto \rho(t)$ are completely positive.

LEMMA 1. The generator in (2.4) can always be written as

$$
\begin{aligned}
L[\rho]= & -i[M, \rho(t)]-\frac{1}{2}(\Gamma \rho+\rho \Gamma) \\
& +\frac{1}{2} \sum_{i, k=1}^{3} v_{i k}\left[2 \sigma_{k} \rho \sigma_{i}-\sigma_{i} \sigma_{k} \rho-\rho \sigma_{i} \sigma_{k}\right],
\end{aligned}
$$

where $v_{i k}=\left\langle a_{i} \mid a_{k}\right\rangle$, with $\left|a_{i}\right\rangle, i=1,2,3$, suitable square-summable complex vectors and the $\sigma_{i}$ are the Pauli matrices.

Proof. With $\sigma_{0}$ the identity $2 \times 2$ matrix, we consider (2.5) and write the (at most countably many) operators $A_{j}$ as $A_{j}=\sum_{\mu=0}^{3} a_{j \mu} \sigma_{\mu}$. Let $\left|a_{\mu}\right\rangle, \mu=0,1,2,3$, be the vectors of components $a_{j \mu}$ : they are square-summable as we assumed $L_{D}[\rho]$ to be a well-defined linear map from the algebra of $2 \times 2$ matrices into itself. Then, (2.5) reads

$$
L_{D}[\rho]=i \sum_{k=1}^{3} \mathcal{I} m\left(v_{0 k}\right)\left[\sigma_{k}, \rho\right]+\frac{1}{2} \sum_{i, k=1}^{3} v_{i k}\left[2 \sigma_{k} \rho \sigma_{i}-\sigma_{i} \sigma_{k} \rho-\rho \sigma_{i} \sigma_{k}\right] .
$$

On the other hand, writing the mass matrix in the Weisskopf-Wigner term of (2.4) as $M=\sum_{\mu=0}^{3} m_{\mu} \sigma_{\mu}$, we get $-i[M, \rho]=-i \sum_{k=1}^{3} m_{k}\left[\sigma_{k}, \rho\right]$.

Thus, we see that the first part of the contribution of the dissipative component $L_{D}[\rho]$ of the generator can be absorbed in a redefinition of the matrix $M$ in (2.3) in such a way that this latter coincides with the phenomenological matrix of physical masses.

Rem ark 1. The dissipative term (second line) of (2.6) is in Kossakowski's form ([1, $4]$ ). Notice that it is now completely separated from the Hamiltonian part (first line). Finally, the $3 \times 3$ matrix

$$
V=\left(\begin{array}{lll}
v_{11} & v_{12} & v_{13} \\
v_{21} & v_{22} & v_{23} \\
v_{31} & v_{32} & v_{33}
\end{array}\right)
$$


where $v_{i j}=\left\langle a_{i} \mid a_{j}\right\rangle=v_{j i}^{*}$, is a positive definite matrix. The latter is also a sufficient condition for the evolution generated by (2.6) to be a quantum dynamical semigroup, that is for the maps $\gamma_{t}: \rho \mapsto \rho(t)$ to be completely positive. Indeed, if $V \geq 0$ then its entries can be always written as scalar products of 3 suitable (square-summable) complex vectors.

Next, as an application of the algebra of the Pauli matrices we have (see [1]):

Lemma 2. Writing $2 \times 2$ density matrices $\rho$ as $\rho=\sum_{\mu=0}^{3} \rho_{\mu} \sigma_{\mu}$, the action $\rho \mapsto L_{D}[\rho]$ amounts to $|\rho\rangle \mapsto \mathcal{L}|\rho\rangle$, where, in vectorial notation, $|\rho\rangle=\left(\rho_{0}, \rho_{1}, \rho_{2}, \rho_{3}\right)$ and

$$
\mathcal{L}=-2\left(\begin{array}{cccc}
0 & 0 & 0 & 0 \\
2 \mathcal{I} m\left(v_{32}\right) & v_{22}+v_{33} & -\mathcal{R} e\left(v_{12}\right) & -\mathcal{R} e\left(v_{13}\right) \\
2 \mathcal{I} m\left(v_{13}\right) & -\mathcal{R} e\left(v_{12}\right) & v_{11}+v_{33} & -\mathcal{R} e\left(v_{23}\right) \\
2 \mathcal{I} m\left(v_{21}\right) & -\mathcal{R} e\left(v_{13}\right) & -\mathcal{R} e\left(v_{23}\right) & v_{11}+v_{22}
\end{array}\right) .
$$

According to the idea that coherence is being lost in the course of time as outlined in the introduction, we want the dissipative non-standard modification $L_{D}[\rho]$ to increase the von Neumann entropy of any initial state.

LEMmA 3. Let $\rho(t):=\exp \left(t L_{D}\right) \rho$, with $\operatorname{Tr}(\rho)=1$ and $\operatorname{Tr}\left(L_{D}[\rho]\right)=0$. Necessary and sufficient condition for the von Neumann entropy $S(\rho(t)):=-\operatorname{Tr}(\rho(t) \log \rho(t))$ not to decrease is that $\mathcal{I} m\left(v_{i j}\right)=0$ for $i \neq j, i=1,2,3$, and that

$$
\mathcal{M}=\left(\begin{array}{ccc}
v_{22}+v_{33} & -v_{12} & -v_{13} \\
-v_{12} & v_{11}+v_{33} & -v_{23} \\
-v_{13} & -v_{23} & v_{11}+v_{22}
\end{array}\right)
$$

be positive definite.

Proof. Let $\rho$ be a kaon state as in $(2.2)$, with $\rho_{0}=1 / 2,1 / 4 \geq|\rho|^{2} \equiv \rho_{1}^{2}+\rho_{2}^{2}+\rho_{3}^{2}$, its eigenvalues, respectively eigenprojections being given by:

$$
\rho_{ \pm}=\frac{1 \pm 2|\rho|}{2}, \quad R_{ \pm}=\frac{1}{2|\rho|}\left(\begin{array}{cc}
|\rho| \pm \rho_{3} & \pm\left(\rho_{1}-i \rho_{2}\right) \\
\pm\left(\rho_{1}+i \rho_{2}\right) & |\rho| \mp \rho_{3}
\end{array}\right) .
$$

Expanding $R_{ \pm}=\left(\sigma_{0} \pm \sum_{i=1}^{3} n_{i} \sigma_{i}\right) / 2$, with $n_{i}=\rho_{i} /|\rho|$, and, by means of $(2.9), L_{D}[\rho]=$ $\sum_{i=1}^{3} \ell_{i} \sigma_{i}$, with $\ell_{i}=\sum_{\mu=0}^{3} \mathcal{L}_{i \mu} \rho_{\mu}$, we obtain

$$
\begin{aligned}
\left.\frac{\mathrm{d} S(\rho(t))}{\mathrm{d} t}\right|_{t=0} & =-\operatorname{Tr}\left(\left.\frac{\mathrm{d} \rho(t)}{\mathrm{d} t}\right|_{t=0} \log \rho\right) \\
& =-\operatorname{Tr}\left(R_{+} L_{D}[\rho]\right) \log \rho_{+}-\operatorname{Tr}\left(R_{-} L_{D}[\rho]\right) \log \rho_{-} \\
& =-\operatorname{Tr}\left(\left(\sum_{i=1}^{3} n_{i} \sigma_{i}\right)\left(\sum_{k=1}^{3} \ell_{k} \sigma_{k}\right)\right) \log \frac{\rho_{+}}{\rho_{-}}=-\left(\sum_{i=1}^{3} n_{i} \ell_{i}\right) \log \frac{\rho_{+}}{\rho_{-}} \\
& =-\frac{1}{|\rho|}\left(\frac{1}{2} \sum_{i=1}^{3} \mathcal{L}_{i 0} \rho_{i}+\sum_{i, j=1}^{3} \mathcal{L}_{i j} \rho_{i} \rho_{j}\right) \log \frac{\rho_{+}}{\rho_{-}} .
\end{aligned}
$$


Let $\rho_{1}=\varepsilon,|\varepsilon| \leq 1 / 2$ and $\rho_{2}=\rho_{3}=0$. Then, using (2.9), (2.12) reads

$$
\left.\frac{\mathrm{d} S(\rho(t))}{\mathrm{d} t}\right|_{t=0}=\frac{2}{|\varepsilon|}\left(\left(v_{22}+v_{33}\right) \varepsilon^{2}+\mathcal{I} m\left(v_{32}\right) \varepsilon\right) \log \frac{\rho_{+}}{\rho_{-}} .
$$

The Schwartz and binomial inequalities imply $\left|\mathcal{I} m\left(v_{32}\right)\right| \leq \sqrt{v_{22} v_{33}} \leq\left(v_{22}+v_{33}\right) / 2$. Then, since $\rho_{+} \geq \rho_{-}$, the right hand side of (2.13) can be always made negative by choosing

$$
\operatorname{sign}(\varepsilon)=-\operatorname{sign}\left(\operatorname{I} m\left(v_{32}\right)\right), \quad|\varepsilon| \leq \frac{\left|\mathcal{I} m\left(v_{32}\right)\right|}{v_{22}+v_{33}},
$$

unless $\operatorname{Im}\left(v_{32}\right)=0$ which implies $v_{23}=\operatorname{R} e\left(v_{23}\right)=v_{23}$.

When $\rho_{1}=\rho_{3}=0, \rho_{2}=\varepsilon$ and $\rho_{1}=\rho_{2}=0, \rho_{3}=\varepsilon$, analogous arguments lead to $\operatorname{Im}\left(v_{21}\right)=\operatorname{I} m\left(v_{13}\right)=0, v_{12}=\mathcal{R} e\left(v_{12}\right)=v_{21}$. Then,

$$
\left.\frac{\mathrm{d} S(\rho(t))}{\mathrm{d} t}\right|_{t=0}=-\frac{1}{|\rho|}\left(\sum_{i, k=1}^{3} \mathcal{L}_{i k} \rho_{i} \rho_{k}\right) \log \frac{\rho_{+}}{\rho_{-}} .
$$

The proof is completed by setting $-2 \mathcal{M}_{i j}=\mathcal{L}_{i j}, i, j=1,2,3$.

Lemma 4. Given the positive definite matrix $V$ in (2.8) with $v_{i j}=v_{j i}$, the matrix $\mathcal{M}$ in (2.10) is positive definite.

Proof. As $V$ is positive definite

$$
\begin{array}{ll}
v_{11} \geq 0, & v_{12}^{2} \leq v_{11} v_{22} \\
v_{22} \geq 0, & v_{13}^{2} \leq v_{11} v_{33} \\
v_{33} \geq 0, & v_{23}^{2} \leq v_{22} v_{33}
\end{array}
$$

and

$$
\operatorname{Det} V=v_{11} v_{22} v_{33}+2 v_{12} v_{23} v_{13}-v_{11} v_{23}^{2}-v_{22} v_{13}^{2}-v_{33} v_{12}^{2} \geq 0 .
$$

From (2.16) it follows $v_{11} v_{22} v_{33} \geq\left|v_{12} v_{23} v_{13}\right|$. Therefore

$$
\begin{aligned}
\operatorname{Det} \mathcal{M} & =\left(v_{22}+v_{33}\right)\left(v_{11}+v_{33}\right)\left(v_{11}+v_{22}\right)-2 v_{12} v_{23} v_{13} \\
& -\left(v_{22}+v_{33}\right) v_{23}^{2}-\left(v_{11}+v_{22}\right) v_{12}^{2}-\left(v_{11}+v_{33}\right) v_{13}^{2} \\
& =2\left(v_{11} v_{22} v_{33}-v_{12} v_{23} v_{13}\right)+v_{11} v_{22}\left(v_{11} v_{22}-v_{12}^{2}\right) \\
& +v_{11} v_{33}\left(v_{11} v_{33}-v_{13}^{2}\right)+v_{22} v_{33}\left(v_{22} v_{33}-v_{23}^{2}\right) \geq 0 .
\end{aligned}
$$

Remark 2. In conclusion, a part from suitable redefinitions of the mass matrix $M$, the most general choice in (2.4-5) ensuring entropy increase is $A_{j}=\sum_{\mu=0}^{3} a_{j \mu} \sigma_{\mu}$, such that

$$
v_{i k}=\sum_{j} a_{j i}^{*} a_{j k}=v_{k i}, \quad \mathcal{I} m\left(v_{i 0}\right)=\sum_{j} \frac{a_{j i}^{*} a_{j 0}-a_{j 0}^{*} a_{j i}}{2 i}=0, i, k=1,2,3 .
$$

With the $A_{j}$ as in the previous Remark, it is convenient to introduce the following parametrization:

$$
\begin{aligned}
& a=v_{22}+v_{33}, \quad b=-v_{12}, \quad c=-v_{13}, \\
& \alpha=v_{11}+v_{33}, \quad \beta=-v_{23}, \\
& \gamma=v_{11}+v_{22} .
\end{aligned}
$$


Then, (2.9) reads

$$
\mathcal{L}=-2\left(\begin{array}{cccc}
0 & 0 & 0 & 0 \\
0 & a & b & c \\
0 & b & \alpha & \beta \\
0 & c & \beta & \gamma
\end{array}\right)
$$

while, the inequalities (necessary and sufficient to complete positivity) are expressed by (compare (2.16-17)):

$$
\begin{array}{ll}
0 \leq a \leq \alpha+\gamma, & 4 b^{2} \leq \gamma^{2}-(a-\alpha)^{2}, \\
0 \leq \alpha \leq a+\gamma, & 4 c^{2} \leq \alpha^{2}-(a-\gamma)^{2}, \\
0 \leq \gamma \leq a+\alpha, & 4 \beta^{2} \leq a^{2}-(\alpha-\gamma)^{2}
\end{array}
$$

and

$$
\begin{aligned}
& \frac{(\alpha+\gamma-a)(a+\gamma-\alpha)(a+\alpha-\gamma)}{8}-2 b c \beta \\
& \quad-\beta^{2} \frac{\alpha+\gamma-a}{2}-c^{2} \frac{a+\gamma-\alpha}{2}-b^{2} \frac{a+\alpha-\gamma}{2} \geq 0 .
\end{aligned}
$$

Remark 3. In $[18,19]$ the proposed non-standard quantum mechanical kaon timeevolution is generated as in (2.4), but the non-standard piece of the generator corresponds to a $4 \times 4$ matrix

$$
\mathcal{L}=-2\left(\begin{array}{cccc}
0 & 0 & 0 & 0 \\
0 & 0 & 0 & 0 \\
0 & 0 & \alpha & \beta \\
0 & 0 & \beta & \gamma
\end{array}\right)
$$

with $\alpha \geq 0, \gamma \geq 0$ and $\alpha \gamma \geq \beta^{2}$. It is easy to check that the inequalities (2.22-23) can be satisfied only if $\alpha=\gamma, \beta=0$. Even such almost trivial non-standard modification seems to be ruled out by the available experimental data that point to $\alpha>>\gamma$.

Thus, the non-standard evolution proposed in $[18,19]$ can only be a very particular instance of complete positive dynamical map: it is more likely to be only positive, failing to be already two-positive ([21]).

3. Comparison with experimental results. The most interesting aspect of Kmeson physics is that the hypothesis of a non-standard dissipative quantum mechanical evolution and its supposedly completely positive character have a chance to be submitted to experimental tests. Before explaining how, it is useful to translate the evolution equation (2.6) in a vectorial way (see Lemma 2) by means of (2.21) and by rewriting the Weisskopf-Wigner term in the generator as a $4 \times 4$ matrix $\mathcal{H}$ acting on the 4 -vector $|\rho(t)\rangle=\left(\rho_{0}(t), \rho_{1}(t), \rho_{2}(t), \rho_{3}(t)\right)$ :

$$
\left.\frac{\partial}{\partial t}|\rho(t)=(\mathcal{H}+\mathcal{L})| \rho(t)\right\rangle .
$$

K-mesons (weakly) interact violating Charge-Parity conservation. The eigenstates of the Weisskopf-Wigner Hamiltonian (2.3) effectively describing such state of affair:

$$
\left|K_{S}\right\rangle=N_{S}\left(\left|K_{1}\right\rangle+\epsilon_{S}\left|K_{2}\right\rangle\right), \quad\left|K_{L}\right\rangle=N_{L}\left(\left|K_{2}\right\rangle+\epsilon_{L}\left|K_{2}\right\rangle\right),
$$


where $N_{S, L}=\left[1+\left|\epsilon_{S, L}\right|^{2}\right]^{-1 / 2}$, cannot coincide with the $C P$-eigenstates $\left|K_{1,2}\right\rangle$ of (2.1). Thus, the complex parameters $\epsilon_{S, L}$ measure the degree of $C P$-violation (and also of $C P T$-violation for $\left.\epsilon_{S} \neq \epsilon_{L}\right)([27])$.

The entries of the Weisskopf-Wigner Hamiltonian can be expressed in terms of the complex parameters $\epsilon_{S}, \epsilon_{L}$, and the four real parameters, $m_{S}, \gamma_{S}$ and $m_{L}, \gamma_{L}$ characterizing the eigenvalues of $H: \lambda_{S, L}=m_{S, L}-\frac{i}{2} \gamma_{S, L}$. Experimentally, the quantities

$$
\Delta \Gamma=\gamma_{S}-\gamma_{L}, \quad \Delta m=m_{L}-m_{S},
$$

corresponding to the differences between decay widths $\left(\gamma_{S}^{-1} \simeq 10^{-10} \mathrm{sec}, \gamma_{L}^{-1} \simeq 10^{-8} \mathrm{sec}\right)$ and masses of the states $K_{S}$ and $K_{L}$, turn out to be positive with $\Delta \Gamma$ of the order of $10^{-14} \mathrm{GeV}$ and $\Delta \Gamma \simeq 2 \Delta \mathrm{m}$. The $C P$-violating parameters $\epsilon_{S, L}$ are of the order $10^{-3}$.

Under physically motivated arguments $([18,19])$ the non-standard phenomenological parameters $a, b, c, \alpha, \beta$ and $\gamma$ are expected to be small (of the order of $\epsilon_{S, L} \Delta \Gamma$ ). One can thus try a perturbative solution of the Schrödinger-like equation (3.1) by distinguishing the various orders of contribution with respect to the small parameters $\epsilon_{S, L}$ in $\mathcal{H}$ :

$$
\mathcal{T}:=\mathcal{H}+\mathcal{L}=\mathcal{H}_{0}+\left(\mathcal{H}_{1}+\mathcal{L}\right)+\mathcal{H}_{2}+\ldots
$$

For later applications it is more convenient to change representation and to write kaon states as 4-vectors $|\rho\rangle=\left(\rho_{1}, \rho_{2}, \rho_{3}, \rho_{4}\right)$ with components from (2.2) where, now,

$$
\rho=\left(\begin{array}{ll}
\rho_{1} & \rho_{3} \\
\rho_{4} & \rho_{2}
\end{array}\right), \quad \rho_{4}=\rho_{3}^{*} .
$$

Then $([20])$

$$
\mathcal{H}_{2}=\left(\begin{array}{cccc}
-\mathcal{R} e\left(\epsilon_{S} \epsilon_{L} \Delta \Gamma_{-}\right) & 0 & 0 & 0 \\
0 & \mathcal{R} e\left(\epsilon_{S} \epsilon_{L} \Delta \Gamma_{-}\right) & 0 & 0 \\
0 & 0 & -i \mathcal{I} m\left(\epsilon_{S} \epsilon_{L} \Delta \Gamma_{-}\right) & 0 \\
0 & 0 & 0 & i \mathcal{I} m\left(\epsilon_{S} \epsilon_{L} \Delta \Gamma_{-}\right)
\end{array}\right)
$$

while the matrix $\mathcal{L}$ takes the form

$$
\mathcal{L}=\left(\begin{array}{cccc}
-\gamma & \gamma & -C & -C^{*} \\
\gamma & -\gamma & C & C^{*} \\
-C^{*} & C^{*} & -A & B \\
-C & C & B^{*} & -A
\end{array}\right)
$$

where we have introduced the convenient notation:

$$
A=\alpha+a, \quad B=\alpha-a+2 i b, \quad C=c+i \beta .
$$


One can now separate the diagonal $\mathcal{H}_{0}$ from the rest and solve (3.1) iteratively. For our purposes, it will be sufficient to keep up to second order contributions ([20]):

$$
\begin{aligned}
& |\rho(t)\rangle=e^{\mathcal{H}_{0} t}|\rho(0)\rangle \\
& +\int_{0}^{t} d s e^{\mathcal{H}_{0}(t-s)}\left[\mathcal{H}_{1}+\mathcal{T}\right] e^{\mathcal{H}_{0} s}|\rho(0)\rangle+\int_{0}^{t} d s e^{\mathcal{H}_{0}(t-s)} \mathcal{H}_{2} e^{\mathcal{H}_{0} s}|\rho(0)\rangle \\
& \quad+\int_{0}^{t} d s_{1} \int_{0}^{s_{1}} d s_{2} e^{\mathcal{H}_{0}\left(t-s_{1}\right)}\left[\mathcal{H}_{1}+\mathcal{T}\right] e^{\mathcal{H}_{0}\left(s_{1}-s_{2}\right)}\left[\mathcal{H}_{1}+\mathcal{T}\right] e^{\mathcal{H}_{0} s_{2}}|\rho(0)\rangle .
\end{aligned}
$$

That is, for a given initial state $\rho$, the components of the solution $|\rho(t)\rangle$ to (3.1) can be approximated by

$$
\rho_{i}(t) \simeq \sum_{j=1}^{4}\left[M_{i j}^{(0)}(t)+M_{i j}^{(1)}(t)+M_{i j}^{(2)}(t)\right] \rho_{j}, \quad i=1,2,3,4 .
$$

The matrix $M^{(0)}$ has only diagonal non-vanishing entries:

$$
\begin{array}{ll}
M_{11}^{(0)}(t)=e^{-\gamma_{S} t}, & M_{33}^{(0)}(t)=e^{-\widetilde{\Gamma}_{-} t}, \\
M_{22}^{(0)}(t)=e^{-\gamma_{L} t}, & M_{44}^{(0)}(t)=e^{-\widetilde{\Gamma}_{+} t},
\end{array}
$$

where

$$
\widetilde{\Gamma}_{ \pm}=\Gamma_{ \pm}+A-\gamma-2 \mathcal{R} e\left[C\left(\epsilon_{S}^{*}-\epsilon_{L}\right)\right] \mp i \frac{|B|^{2}}{2 \Delta m} \mp 8 i \Delta m\left|\frac{C}{\Delta \Gamma_{+}}\right|^{2}
$$

and

$$
\Gamma_{ \pm}=\Gamma \pm i \Delta m, \quad \Delta \Gamma_{ \pm}=\Delta \Gamma \pm 2 i \Delta m, \quad \Gamma=\frac{\gamma_{S}+\gamma_{L}}{2} .
$$

The entries of $M^{(1)}, M^{(2)}$ contain first, second order terms in the small parameters $([20])$. One can also extract the contributions $\rho_{L}$ and $\rho_{S}$ that correspond to the $K_{L}$ and $K_{S}$ neutral kaons:

$$
\rho_{L}=\left[\begin{array}{cc}
\left|\epsilon_{L}+\frac{2 C^{*}}{\Delta \Gamma_{-}}\right|^{2}+\frac{\gamma}{\Delta \Gamma}-8\left|\frac{C}{\Delta \Gamma_{+}}\right|^{2}-4 \mathcal{R} e\left(\frac{\epsilon_{L} C}{\Delta \Gamma}\right) & \epsilon_{L}+\frac{2 C^{*}}{\Delta \Gamma_{-}} \\
\epsilon_{L}^{*}+\frac{2 C}{\Delta \Gamma_{+}} & 1
\end{array}\right],
$$

and

$$
\rho_{S}=\left[\begin{array}{c}
1 \\
\epsilon_{S}^{*}+\frac{2 C^{*}}{\Delta \Gamma_{+}} \\
\epsilon_{S}+\frac{2 C}{\Delta \Gamma_{-}} \\
\left|\epsilon_{S}+\frac{2 C}{\Delta \Gamma_{-}}\right|^{2}-\frac{\gamma}{\Delta \Gamma}-8\left|\frac{C}{\Delta \Gamma_{+}}\right|^{2}-4 \mathcal{R} e\left(\frac{\epsilon_{S} C^{*}}{\Delta \Gamma}\right)
\end{array}\right] .
$$

Remark 4. When the parameters $a, b, c, \alpha, \beta$ and $\gamma$ vanish, the above two states coincide, as they should, with the projections $\rho_{L, S}$ onto the eigenstates $(3.2)\left|K_{L, S}\right\rangle$ of the Weisskopf-Wigner Hamiltonian (2.3). The subscripts $(L, S)$ refer to the very different life-times of the physical states $K_{L, S}$.

The link between the preceding abstract theoretical setting with concrete experiments is given by the fact that what experimentalists actually measure are certain typical observables for the $K^{0}-\overline{K^{0}}$ system. Indeed, any physical property of the neutral kaons 
can be extracted from the density matrix $\rho(t)$ by taking its trace with suitable hermitian operators.

Useful observables are associated with the fact that, with certain probabilities, neutral kaons decay into other elementary particles as pions $\pi^{ \pm}, \pi^{0}$, or pions, leptons (electrons or muons) and neutrinos (semileptonic decays $K \mapsto \pi \ell \nu$ ).

The amplitudes for the decay of a $K^{0}$ state into $\pi^{+} \pi^{-}$and $\pi^{0} \pi^{0}$ final states are usually parametrized as follows, in terms of the $s$-wave phase-shifts $\delta_{i}$ and the complex coefficients $A_{i}, B_{i}, i=1,2$ ([27]):

$$
\begin{aligned}
& \mathcal{A}\left(K^{0} \rightarrow \pi^{+} \pi^{-}\right)=\left(A_{0}+B_{0}\right) e^{i \delta_{0}}+\frac{1}{\sqrt{2}}\left(A_{2}+B_{2}\right) e^{i \delta_{2}}, \\
& \mathcal{A}\left(K^{0} \rightarrow \pi^{0} \pi^{0}\right)=\left(A_{0}+B_{0}\right) e^{i \delta_{0}}-\sqrt{2}\left(A_{2}+B_{2}\right) e^{i \delta_{2}}
\end{aligned}
$$

where the indices 0,2 refers to the total isospin $I$. The amplitudes for the $\overline{K^{0}}$ decays are obtained from these with the substitutions: $A_{i} \rightarrow A_{i}^{*}$ and $B_{i} \rightarrow-B_{i}^{*}$. The imaginary parts of $A_{i}$ signals direct $C P$-violation, while a non zero value for $B_{i}$ will also break $C P T$ invariance.

The operators that describe the decays $K \mapsto \pi^{+} \pi^{-}$and $K \mapsto \pi^{0} \pi^{0}$ can be written as:

$$
\begin{aligned}
& \mathcal{O}_{+-}=\left|X_{+-}\right|^{2}\left[\begin{array}{cc}
1 & Y_{+-} \\
Y_{+-}^{*} & \left|Y_{+-}\right|^{2}
\end{array}\right], \\
& \mathcal{O}_{00}=\left|X_{00}\right|^{2}\left[\begin{array}{cc}
1 & Y_{00} \\
Y_{00}^{*} & \left|Y_{00}\right|^{2}
\end{array}\right],
\end{aligned}
$$

where the complex parameters $X$ and $Y$ can be expressed in terms of $A_{i}, B_{i}$ and $\delta_{i}$ :

$$
\begin{gathered}
X_{+-}=\sqrt{2}\left[\mathcal{R} e\left(A_{0}\right)+i \mathcal{I} m\left(B_{0}\right)\right] e^{i \delta_{0}}+\left[\mathcal{R} e\left(A_{2}\right)+i \mathcal{I} m\left(B_{2}\right)\right] e^{i \delta_{2}}, \\
X_{00}=\sqrt{2}\left[\mathcal{R} e\left(A_{0}\right)+i \mathcal{I} m\left(B_{0}\right)\right] e^{i \delta_{0}}-2\left[\mathcal{R} e\left(A_{2}\right)+i \mathcal{I} m\left(B_{2}\right)\right] e^{i \delta_{2}}, \\
Y_{+-}=\varepsilon-\epsilon_{L}+\varepsilon^{\prime}, \quad Y_{00}=\varepsilon-\epsilon_{L}-2 \varepsilon^{\prime},
\end{gathered}
$$

where

$$
\begin{gathered}
\varepsilon=\left[\frac{\epsilon_{L}+\epsilon_{S}}{2}+i \frac{\mathcal{I} m\left(A_{0}\right)}{\mathcal{R} e\left(A_{0}\right)}\right]+\left[\frac{\epsilon_{L}-\epsilon_{S}}{2}+\frac{\mathcal{R} e\left(B_{0}\right)}{\mathcal{R} e\left(A_{0}\right)}\right], \\
\varepsilon^{\prime}=\frac{i e^{i\left(\delta_{2}-\delta_{0}\right)}}{\sqrt{2}} \frac{\mathcal{R} e\left(A_{2}\right)}{\mathcal{R} e\left(A_{0}\right)}\left[\frac{\mathcal{I} m\left(A_{2}\right)}{\mathcal{R} e\left(A_{2}\right)}-\frac{\mathcal{I} m\left(A_{0}\right)}{\mathcal{R} e\left(A_{0}\right)}\right]+i\left[\frac{\mathcal{R} e\left(B_{0}\right)}{\mathcal{R} e\left(A_{0}\right)}-\frac{\mathcal{R} e\left(B_{2}\right)}{\mathcal{R} e\left(A_{0}\right)}\right] .
\end{gathered}
$$

By noticing that:

$$
\operatorname{Tr}\left(\mathcal{O}_{+-} \rho_{L}\right) \equiv\left|\left\langle\pi^{+} \pi^{-}|T| K_{L}\right\rangle\right|^{2}
$$

one easily obtains the corresponding decay rates for the physical states $K_{L}$ and $K_{S}([23])$ :

$$
\begin{aligned}
& \left|\mathcal{A}\left(K_{L} \rightarrow \pi^{+} \pi^{-}\right)\right|^{2} \equiv \operatorname{Tr}\left(\mathcal{O}_{+-} \rho_{L}\right)=\left|X_{+-}\right|^{2}\left|N_{L}\right|^{2}\left|\epsilon_{L}+Y_{+-}\right|^{2}, \\
& \left|\mathcal{A}\left(K_{S} \rightarrow \pi^{+} \pi^{-}\right)\right|^{2} \equiv \operatorname{Tr}\left(\mathcal{O}_{+-} \rho_{S}\right)=\left|X_{+-}\right|^{2}\left|N_{S}\right|^{2}\left|1+\epsilon_{S} Y_{+-}\right|^{2} .
\end{aligned}
$$

From this result, one recovers, to leading order in $C P$ and $C P T$ violation and $\Delta I=1 / 2$ enhancement, the familiar expressions ([27]):

$$
\left|\frac{\mathcal{A}\left(K_{L} \rightarrow \pi^{+} \pi^{-}\right)}{\mathcal{A}\left(K_{S} \rightarrow \pi^{+} \pi^{-}\right)}\right|^{2} \simeq\left|\varepsilon+\varepsilon^{\prime}\right|^{2},
$$




$$
\left|\frac{\mathcal{A}\left(K_{L} \rightarrow 2 \pi^{0}\right)}{\mathcal{A}\left(K_{S} \rightarrow 2 \pi^{0}\right)}\right|^{2} \simeq\left|\varepsilon-2 \varepsilon^{\prime}\right|^{2} .
$$

Similar results hold for the matrices $\mathcal{O}_{\pi^{+} \pi^{-} \pi^{0}}, \mathcal{O}_{3 \pi^{0}}, \mathcal{O}_{\ell^{-}}$and $\mathcal{O}_{\ell^{+}}$that describe the decays into $\pi^{+} \pi^{-} \pi^{0}, 3 \pi^{0}, \pi^{+} \ell^{-} \bar{\nu}$ and $\pi^{-} \ell^{+} \nu$.

With the help of these matrices, one can now compute the time-dependence of various useful observables of the neutral kaon system. For example, in the case of charged pions, the decay rate for pure $K^{0}$ initial state is:

$$
\begin{aligned}
R_{+-}(t) & =\frac{\operatorname{Tr}\left[\rho_{K^{0}}(t) \mathcal{O}_{+-}\right]}{\operatorname{Tr}\left[\rho_{K^{0}}(0) \mathcal{O}_{+-}\right]} \\
& =e^{-\gamma_{S} t}+R_{+-}^{L} e^{-\gamma_{L} t}+2 e^{-\Gamma t}\left|\eta_{+-}\right| \cos \left(\Delta m t-\phi_{+-}\right),
\end{aligned}
$$

where $R_{+-}^{L}$ is the two-pion decay rate for the $K_{L}$ state:

$$
R_{+-}^{L}=\left|\epsilon_{L}+\frac{2 C^{*}}{\Delta \Gamma_{-}}+Y_{+-}\right|^{2}+\frac{\gamma}{\Delta \Gamma}-8\left|\frac{C}{\Delta \Gamma_{+}}\right|^{2}-4 \mathcal{R} e\left(\frac{\epsilon_{L} C}{\Delta \Gamma}\right)
$$

while the interference term is determined by the combination

$$
\epsilon_{L}-\frac{2 C^{*}}{\Delta \Gamma_{-}}+Y_{+-} \equiv \eta_{+-}=\left|\eta_{+-}\right| e^{i \phi_{+-}} .
$$

Similar formulas hold for the decay of a $K^{0}$ into neutral pions: one just needs to substitute the index +- with 00 .

Other interesting observables, directly measured in experiments, are the asymmetries associated with the decay into the final state $f$ of an initial $K^{0}$ as compared to the corresponding decay into $\bar{f}$ of an initial $\overline{K^{0}}$. All these asymmetries have the general form

$$
A(t)=\frac{\operatorname{Tr}\left[\rho_{\bar{K}^{0}}(t) \mathcal{O}_{\bar{f}}\right]-\left[\rho_{K^{0}}(t) \mathcal{O}_{f}\right]}{\operatorname{Tr}\left[\rho_{\bar{K}^{0}}(t) \mathcal{O}_{\bar{f}}\right]+\left[\rho_{K^{0}}(t) \mathcal{O}_{f}\right]}
$$

In the case of the $\pi^{+} \pi^{-} \pi^{0}$ final state one finds

$$
\begin{aligned}
A_{+-0}(t)=2 \mathcal{R} e & \left(\epsilon_{S}-\frac{2 C}{\Delta \Gamma_{-}}\right) \\
& -2 e^{-\Delta \Gamma t / 2}\left[\mathcal{R} e\left(\eta_{+-0}\right) \cos (\Delta m t)-\mathcal{I} m\left(\eta_{+-0}\right) \sin (\Delta m t)\right],
\end{aligned}
$$

where

$$
\eta_{+-0} \equiv \epsilon_{S}-\frac{2 C}{\Delta \Gamma_{-}}+Y_{+-0} .
$$

A useful observable involving semileptonic decays is the so called CP-violating charge asymmetry:

$$
\delta(t)=\frac{\operatorname{Tr}\left[\rho(t)\left(\mathcal{O}_{\ell^{+}}-\mathcal{O}_{\ell^{-}}\right)\right]}{\operatorname{Tr}\left[\rho(t)\left(\mathcal{O}_{\ell^{+}}+\mathcal{O}_{\ell^{-}}\right)\right]}
$$

For a state which is initially a pure $K^{0}$, it can be explicitly written as:

$$
\delta(t)=\frac{\delta_{S} e^{-\gamma_{S} t}+\delta_{L} e^{-\gamma_{L} t}+2 e^{-\Gamma t} \cos (\Delta m t)}{e^{-\gamma_{S} t}+e^{-\gamma_{L} t}},
$$


where

$$
\delta_{S}=2 \mathcal{R} e\left(\epsilon_{S}+\frac{2 C}{\Delta \Gamma_{-}}\right), \quad \delta_{L}=2 \mathcal{R} e\left(\epsilon_{L}+\frac{2 C^{*}}{\Delta \Gamma_{-}}\right)
$$

are the charge asymmetries for the $K_{S}$ and $K_{L}$ states.

Remark 5. Notice that the standard quantum mechanical results, namely those deduced according to the time-evolution generated by the Weisskopf-Wigner Hamiltonian (2.3), can be recovered by putting the non-standard parameters (3.8) all equal to zero.

The phenomenological quantities $\eta_{+-}, \eta_{+-0}, \delta_{L}, \delta_{S}$ are directly accessible to the experiment. Using the most recent data, one can obtain estimates for some of the nonstandard parameters:

$$
\begin{aligned}
& a=(2.3 \pm 2.9) \times 10^{-17} \mathrm{GeV}, \\
& c=(1.0 \pm 1.4) \times 10^{-17} \mathrm{GeV}, \\
& \alpha=(2.1 \pm 2.9) \times 10^{-17} \mathrm{GeV}, \\
& \beta=(-1.0 \pm 1.5) \times 10^{-17} \mathrm{GeV}, \\
& \gamma=(0.1 \pm 30.2) \times 10^{-20} \mathrm{GeV} .
\end{aligned}
$$

Unfortunately the precision of the present experimental data on the neutral kaon system is not high enough to allow a meaningful test of the inequalities (2.22-23) and hence of the hypothesis of complete positivity. However, the next-generation experiments should provide more complete and precise data. In this respect, particularly promising are the planned experiments on correlated neutral kaons at $\phi$-factories.

4. Entangled kaons. The meson $\phi$ is a spin 1 elementary particle which can decay into two neutral (spinless) kaons flying apart in opposite directions. The only way to do that without violating conservation laws is via a spatially antisymmetric state; in the $\phi$-particle rest frame:

$$
\left|\psi_{A}\right\rangle=\frac{1}{\sqrt{2}}\left(\left|K_{1},-p\right\rangle \otimes\left|K_{2}, p\right\rangle-\left|K_{2},-p\right\rangle \otimes\left|K_{1}, p\right\rangle\right) .
$$

The corresponding density operator $\rho_{A}$ can be represented by the $4 \times 4$ matrix

$$
\rho_{A}=\frac{1}{2}\left[P_{1} \otimes P_{2}+P_{2} \otimes P_{1}-P_{3} \otimes P_{4}-P_{4} \otimes P_{3}\right]
$$

in terms of the single kaon projectors:

$$
P_{1} \equiv\left|K_{1}\right\rangle\left\langle K_{1}\left|=\left(\begin{array}{ll}
1 & 0 \\
0 & 0
\end{array}\right), \quad P_{2} \equiv\right| K_{2}\right\rangle\left\langle K_{2}\right|=\left(\begin{array}{ll}
0 & 0 \\
0 & 1
\end{array}\right)
$$

and of the off-diagonal operators

$$
P_{3} \equiv\left|K_{1}\right\rangle\left\langle K_{2}\left|=\left(\begin{array}{ll}
0 & 1 \\
0 & 0
\end{array}\right), \quad P_{4} \equiv\right| K_{2}\right\rangle\left\langle K_{1}\right|=\left(\begin{array}{ll}
0 & 0 \\
1 & 0
\end{array}\right) .
$$

Remark 6. In the standard quantum mechanical picture the time-evolution of the two correlated neutral kaons produced in $\phi$-decays is just the tensor product of the single-kaon time-evolutions generated by the effective Weisskopf-Wigner Hamilto- 
nian (2.3). Even in presence of an environment, the most natural extension is to assume that, once produced in a $\phi$ decay, the two kaons evolve independently in time each with the (single-kaon) completely positive map $\gamma_{t}$ generated by $(2.6)$.

According to the previous Remark, the density matrix that describes a situation in which the first kaon has evolved up to proper time $\tau_{1}$ and the second up to proper time $\tau_{2}$ is given by:

$$
\begin{aligned}
\rho_{A}\left(\tau_{1}, \tau_{2}\right) \equiv & \left(\gamma_{\tau_{1}} \otimes \gamma_{\tau_{2}}\right)\left[\rho_{A}\right] \\
= & \frac{1}{2}\left[P_{1}\left(\tau_{1}\right) \otimes P_{2}\left(\tau_{2}\right)+P_{2}\left(\tau_{1}\right) \otimes P_{1}\left(\tau_{2}\right)\right. \\
& \left.\quad-P_{3}\left(\tau_{1}\right) \otimes P_{4}\left(\tau_{2}\right)-P_{4}\left(\tau_{1}\right) \otimes P_{3}\left(\tau_{2}\right)\right] .
\end{aligned}
$$

The typical observables that can be studied in such quantum interferometer are double decay rates, i.e. the probabilities that a kaon decays into a final state $f_{1}$ at proper time $\tau_{1}$, while the other kaon decays into the final state $f_{2}$ at proper time $\tau_{2}([26])$ :

$$
\mathcal{G}\left(f_{1}, \tau_{1} ; f_{2}, \tau_{2}\right) \equiv \operatorname{Tr}\left[\left(\mathcal{O}_{f_{1}} \otimes \mathcal{O}_{f_{2}}\right) \rho_{A}\left(\tau_{1}, \tau_{2}\right)\right]
$$

For example, in the case of the $\pi^{+} \pi^{-}$final states, at equal decay times $\tau_{1}=\tau_{2}=\tau$ :

$$
\begin{array}{r}
\mathcal{G}\left(\pi^{+} \pi^{-}, \tau ; \pi^{+} \pi^{-}, \tau\right)=\left|X_{+-}\right|^{4} e^{-\gamma_{S} \tau}\left\{e^{-\gamma_{L} \tau}\left(R_{+-}^{L}-\left|\eta_{+-}\right|^{2}\right)\right. \\
-e^{-\gamma_{S} \tau}\left[\frac{\gamma}{\Delta \Gamma}+8\left|\frac{C}{\Delta \Gamma_{+}}\right|^{2}-4 \mathcal{R} e\left(\frac{\epsilon_{L} C}{\Delta \Gamma}\right)\right] \\
\left.-e^{-\Gamma \tau} 8 \mathcal{R} e\left(\frac{\eta_{+-} C}{\Delta \Gamma_{+}} e^{-i \Delta m \tau}\right)\right\} .
\end{array}
$$

This time-behaviour is completely different from the one required by ordinary quantum mechanics, which predicts:

$$
\mathcal{G}(f, \tau ; f, \tau) \equiv 0
$$

Therefore, by studying double decay rates in a high-luminosity $\phi$-factory it will be possible to determine the values of the non-standard parameres $a, b, c, \alpha, \beta, \gamma$, and as a consequence test the hypothesis of complete positivity of the (single) kaon time-evolution.

We conclude this contribution by pointing out another unique feature of the $\phi$ interferometry physics. In fact, it appears that the question outlined in the introduction, whether complete positivity is just a reasonable technical, but physically not necessary, request, can be brought down to actual experimental situations.

The abstractness and triviality of the coupling with an $N$-level system leading to the notion of complete positivity becomes concretely physical when the other system is, as discussed above, another kaon evolving in time exactly as the previous one.

Had we used a simply positive time-evolution as in [18, 19] (see Remark 4), inconsistencies in the formalism would have emerged. In fact, one of the properties of the density matrix $\rho_{A}(\tau) \equiv \rho_{A}(\tau, \tau)$ in (4.5) is that its mean value on any vector is positive for all times. Without this basic requirement, the standard probability interpretation of $\rho_{A}(\tau)$ as a state of the correlated kaons would be meaningless. Otherwise one would have to cope with the presence of meaningless negative "probabilities". 
In the case of the vector:

$$
|u\rangle=\frac{1}{\sqrt{2}}\left[\left(\begin{array}{l}
1 \\
0
\end{array}\right) \otimes\left(\begin{array}{l}
1 \\
0
\end{array}\right)+\left(\begin{array}{l}
0 \\
1
\end{array}\right) \otimes\left(\begin{array}{l}
0 \\
1
\end{array}\right)\right],
$$

the complete positivity of the single-kaon dynamics gives, to first order in the small parameters:

$$
\begin{aligned}
\mathcal{U}(\tau) \equiv & \left\langle u\left|\rho_{A}(\tau)\right| u\right\rangle=\left\{\frac{\gamma}{2 \Delta \Gamma}\left(e^{-2 \gamma_{L} \tau}-e^{-2 \gamma_{S} \tau}\right)\right. \\
& \left.-e^{-2 \Gamma \tau}\left[\frac{\alpha-a}{2 \Delta m} \sin (2 \Delta m \tau)+\frac{b}{\Delta m}(\cos (2 \Delta m \tau)-1)\right]\right\} .
\end{aligned}
$$

This result is indeed positive for all times, and vanish only at $\tau=0$, due to the antisymmetry of $\rho_{A}$ in (4.2).

This conclusion is not true in general. When $\tau \rightarrow 0$, one finds

$$
\mathcal{U}(\tau) \simeq(\gamma-\alpha+a) \tau .
$$

If the single kaon dynamics is completely positive, the positivity of (4.10) is guaranteed by the inequalities $(2.22)$, whereas the latter quantity might become negative if we are as in $[18,19]$ : in fact, according to Remark 3 , in this case $a=0$, while $\alpha>>\gamma$ as suggested by experimental data (for a more general treatment of this point see [21]).

A $\phi$-factory, being a high performance quantum interferometer, can measure, at least in principle, observables like (4.9) and therefore further clarify the request of complete positivity for the dynamics of neutral kaons.

Remark 7. The standard quantum mechanical time-evolution of single kaons is automatically completely positive. Thus, no inconsistencies may arise when using the tensor product for the time-evolution of two kaons.

On the contrary, when dealing with kaons as open quantum systems, complete positivity comes as a necessary consequence if we assume that, like in the standard description, also the time evolution of entangled kaons be factorized.

Such an assumption is not only natural: without it, it would be very hard to provide a consistent independent characterization of single kaons. Indeed, if the multi-particle dynamics is not of factorized form, when singling out the evolution of one single particle by tracing away the unwanted degrees of freedom of all other particles, memory effects would unavoidably survive. The latter would then make the single particle description depend on the presence of other particles even if they do not directly influence each other.

From a more general point of view the preceding discussion raises the theoretical question how to consistently describe many-particle systems interacting with an external environment. To our knowledge very little has been done so far on this difficult topic.

\section{References}

[1] R. Alicki and K. Lendi, Quantum Dynamical Semigroups and Applications, Lecture Notes in Phys. 286, Springer-Verlag, Berlin, 1987.

[2] H. Spohn, Rev. Mod. Phys. 53 (1980), 569. 
[3] E. B. Davies, Quantum Theory of Open Systems, Academic Press, New York, 1976.

[4] V. Gorini, A. Frigerio, M. Verri, A. Kossakowski and E. C. G. Surdarshan, Rep. Math. Phys. 13 (1978), 149.

[5] M. Takesaki, Theory of Operator Algebras I, Springer, Berlin, 1979.

6] K. Kraus, States, Effects, and Operations, Lecture Notes in Phys. 190, Springer Verlag, Berlin, 1983.

[7] V. Gorini, A. Kossakowski and E. C. G. Sudarshan, J. Math. Phys. 17 (1976), 821.

[8] G. Lindblad, Comm. Math. Phys. 48 (1976), 119.

[9] P. Pechukas, Phys. Lett. 73 (1994), 1060.

[10] R. Alicki, Phys. Lett. 75 (1995), 3020.

[11] A. Peres, Separability criterion for density matrices, preprint, quant-ph/9604005.

[12] M. Horodecki, P. Horodecki and R. Horodecki, Separability of mixed states: necessary and sufficient conditions, preprint, quant-ph/9605038.

[13] T. D. Lee and C.S. Wu, Ann. Rev. Nucl. Sci. 16 (1966), 511.

[14] S. Hawking, Comm. Math. Phys. 87 (1983), 395.

[15] W. Unruh and R.M. Wald, Phys. Rev. D 52 (1995), 2176.

[16] M. Srednicki, Nucl. Phys. B 410 (1993), 143.

[17] T. Banks, L. Susskind and M. E. Peskin, Nucl. Phys. B 244 (1984), 125.

[18] J. Ellis, J. S. Hagelin, D. V. Nanopoulos and M. Srednicki, Nucl Phys. B 241 (1984), 381. J. Ellis, J. L. Lopez, N. E. Mavromatos and D. V. Nanopolous, Phys. Rev. D 53 (1996), 3846.

[19] P. Huet and M.E. Peskin, Nucl. Phys. B 434 (1995), 3.

[20] F. Benatti and R. Floreanini, Nucl. Phys. B 488 (1997), 335.

[21] F. Benatti and R. Floreanini, Mod. Phys. Lett. A12 (1997), 465.

[22] F. Benatti and R. Floreanini, Completely positive dynamics of correlated neutral kaons, Trieste-preprint, 1997.

[23] F. Benatti and R. Floreanini, Phys. Lett. B 401 (1997), 337.

[24] L. Fonda, G. C. Ghirardi and A. Rimini, Rep. Prog. Phys. 41 (1978), 587.

[25] G. C. Ghirardi, A. Rimini and T. Weber, Phys. Rev. D 34 (1986), 470.

[26] C. D. Buchanan, R. Cousins, C. Dib, R. D. Peccei and J. Quackenbush, Phys. Rev. D 45 (1992), 4088.

[27] L. Maiani, CP and CPT violation in neutral kaon decays, in: The Second Da $\phi$ ne Physics Handbook, L. Maiani, G. Pancheri and N. Paver (eds.), INFN, Frascati, 1995. 